\title{
Schizophrenia Between the Dilemmas of Conceptual Framing
}

\section{Dania-Andreea Radu, Ilinca Untu}

Dania Andreea Radu - M.D., psychiatrist, Ph.D. Student, "Socola” Institute of Psychiatry, Iaşi, Romania, Grigore T.Popa University of Medicine and Pharmacy, Iași, Romania

Ilinca Untu - M.D., Psychiatrist, Ph.D. Student, "Socola” Institute of Psychiatry, Iaşi, Romania, Grigore T.Popa University of Medicine and Pharmacy, Iași, Romania

The term "schizophrenia" dates back about 100 years. Before the valid name was established today, this disorder, as a symptomatic group, was defined by Emil Kraepelin in 1887 -dementia praecox. Despite the absence of an early nosological classification, schizophrenia has been a medical and social reality that has accompanied the whole history of mankind from its beginnings to the present. In ancient Egypt, a series of thought disorders were foreshadowed which overlap in part with what is nowadays the positive, negative and cognitive symptoms of schizophrenia, but the pathological psychiatric manifestations passing as heart disorders, considered as the seat of the spirit. In ancient Greece and Rome, there is the social perception of mental illness without framing what it means to be a series of benchmarks that differentiate individual nosological entities. In the paleo-christian and medieval period, all persons considered "abnormal", either as a result of mental illness, mental retardation or physical deformities, were treated in the same way. Early theories assumed that mental disorders were caused by the evil possession of the body, and the proper treatment was then to exorcise these demons, through various means, from harmless treatments, such as exposing the patient to certain types of music, to some dangerous and sometimes deadly, such as be the release of evil spirits by making holes in the patient's skull.

In the 19th century, Benedict Morel launched the term demence precoce which defined a multitude of symptoms that overlap today with the positive elements of schizophrenia. Shortly, Emil Kraepelin draws a concrete differentiation between dementia praecox and manic depression, thus achieving a conceptual delimitation of two major psychiatric disorders. Dementia praecox is thus considered to be a suffering of the brain which involves also deterioration of cognitive function. 


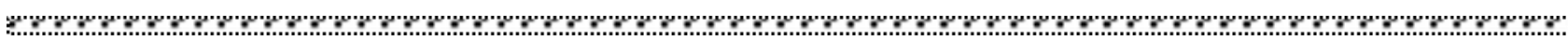

Kraepelin distinguishes three main forms of this disorder - paranoid, disorganized and catatonic. Eugen Bleuler replaced in literature the preceding term with that of SCHIZOPHRENIA, thus defining the schism among thoughts, emotions and behavioral reactions (clinical features that include those exposed by Kraepelin delusional ideas and hallucinations). This is also the first to describe the symptoms of schizophrenia as positive and negative. By changing the name of this major psychiatric disorder, the confusion promoted by the term introduced by Kraepelin is removed and it is established that schizophrenia exists without cognitive impairment and that schizophrenia does not belong to the same diagnostic spectrum with dementia. However, the new term, meant to dissolve any confusion about the nature of the disease, leads to the overlap of schizophrenia, in the collective mind, on the split of personality (multiple personality).

Over the years, Wirsch has analyzed the intuitive classification of the schizophrenia patient, according to whom "the praecox feeling" has nothing to do with mimicry and pantomime, nor with the poor emotional contact of schizophrenia, but with a certain way of being - daseinsweise - a certain way of being in the world and belonging to it. $\mathrm{He}$ argued that this intuitive recognition of schizophrenia is not about guessing or supposing but about being absolutely sure. Muller-Suur argues that the intuitive perception of the elements of schizophrenia does not involve understanding vague, inaccurate elements, but a definite incomprehensibility. Thus a change of perception emerges from being in the world to a specific, individual but incomprehensible order. What is incomprehensible, but perceived intuitively as something well defined, are basic structures of our being that are changed, such as temporality, spatiality and interaction with others. After 100 years of studies, schizophrenia remains a chronic disorder, with a still inaccurate nosological classification. Professor Pull argues that there are no pathognomonic symptoms, with patients with the same diagnosis having different forms of disease manifestation. Although there are standardized interviews and operational definitions, schizophrenia resembles their Babel tower, some of them (eg DSM criteria) confronting practitioners with equivocal standardized diagnostic elements. The lack of stable boundaries between schizophrenia and manicdepressive psychosis, has led to the emergence of an intermediate nosological entity, which borrows the fundamental symptoms of both - schizoaffective disorder.

The thinking of American psychiatrists, which was dominated until the immediate post-World War II period, by the terminology and thinking promoted by Freud's psychoanalysis. In the 1960s, most American psychiatrists abdicated from the Freudian ideological current, as a reaction to the lack of therapeutic response to its methods and to avoid removing psychiatry outside the medical field. In addition, Spitzer and others have relied on the biological approach to mental disorders.

In the 1970s, Gerald Klerman introduced the term neo-Kraepelian, created in a manifesto in which he listed nine claims that led to a new diagnostic system in psychiatry for 16 major psychiatric disorders, his work becoming one of the most cited in psychiatry. This manifesto claims that psychiatry is a real branch of medicine that should use modern scientific methods and base their practice on scientific 
s

evidence. At the same time, he states that psychiatry treats people who are ill and need treatment for mental illness and that there is a border between sick and normal, and mental illnesses are not myths, even if there are mental disorders with subtle clinical manifestation. Mental illnesses are not myths. There are several mental disorders, not a single mental illness that brings together all the psychopathological manifestations. It is the task of psychiatry, as in other medical specialties, to investigate the causes, diagnosis and treatment of mental illness. The orientation of the psychiatrists must be channeled predominantly on the biological coordinate, imposing a real preoccupation with the diagnostic and classification criteria. The diagnostic criteria must be codified, and the criteria must be validated by appropriate scientific methods. Further, medical schools should promote these criteria and not impair them, as has long been the case, improving the relevance and validity of diagnoses by requiring the use of statistical techniques.

In the trend of minimizing the role of psychiatry, R D Laing, a Scottish psychiatrist, along with David Cooper and Thomas Szasz developed the concept of "antipsychiatry", Laing being against TEC and the new antipsychotics and arguing that schizophrenia is "a theory not a fact". Szasz has stated throughout his career that mental illness is a metaphor for human life problems and that mental illness is not a "disease" in the true sense; "There are no biological or chemical tests nor biopsy or necropsy findings for verifying DSM diagnoses." The term has become widely used to refer to a variety of criticisms of psychiatry and the concept of mental illness. In fact, "antipsychiatry" had an important contribution, today the "treatment" being a "bio-psycho-social" and has contributed to the reintegration of patients with schizophrenia into a society that until recently institutionalized and marginalized them. DSM III (1980) has remained in history as the most influential edition of DSM, appearing as a reaction to the request of health insurance companies, to have clear diagnoses and implicit criteria (Previous Research Diagnostic Criteria and DSM III, were used benchmarks vague, inaccurate diagnoses). Since 1980, almost all the studies conducted have been linked to the same diagnostic algorithm.

The history of schizophrenia research overlaps the chronology of recurring themes - several promising clues that have been abandoned and a series of explanatory models, never fully confirmed, but never denied. Most contemporary research ideas are rediscovered early observations and hypotheses (the first decades following Kraepelin's nosological classification). Numerous studies, however small in scope, of an anatomical-pathological nature, have shown the presence of disturbances that especially affect the limbic region and do not appear as an evolutionary degenerative process, but the result of an evolutionary disturbance in the perinatal period. Epidemiological data related to schizophrenia are relevant depending on the issue of comparability of diagnosis over time.

Today's reality calls for schizophrenia to reach more than 23 million people worldwide, but it is not as common as other mental disorders. World statistics show that it is more common in men than women (12 million vs. 9 million) (1.4 X more common in men than women), being associated with considerable disabilities and interferes with socio-professional performance. People with schizophrenia are 2-3 times more 
likely to die at an earlier age than the general population, especially on the basis of cardiovascular, metabolic and infectious diseases. The stigmatization, discrimination and violation of human rights continue to be major obstacles to the socio-professional and educational integration of people with schizophrenia. More than $50 \%$ of people with schizophrenia do not receive adequate care. In low-income countries, 90\% of people with schizophrenia are subtracted. Reduced access to health services is the main reason for this situation. At the same time, people with schizophrenia are much less willing to seek help on their own, compared to the general population.

Schizophrenia remains an undeniable major psychiatric disorder as a clinical reality, but controversial, which still holds a series of questions regarding the correctness of the diagnostic criteria, related to the etiopathogenesis, as well as its multiple implications. The answers are not easy to give, especially since they are, in turn, dependent on other questions that lead to them, in a whirlwind of philosophical, medical, sociological and political dilemmas. What is a mental illness? What does treatment mean? Is treatment necessary? What does it mean to be a psychiatrist? And what does it mean to be patient? To what extent are the definitions of the concepts of "disease" and "treatment" related to a political system, a certain social order? To what extent is medicine itself dependent on power regimes? What is the mind, be it "healthy" or "sick"? What does it mean to be schizophrenic when schizophrenia is a so-called "functional" condition, in other words a disease without an obvious anatomical basis, whose pathology is not linked (at least for now) to any specific objective evidence. 